\title{
Atuação do médico cirurgião de mão em microcirurgia no Brasil ${ }^{*}$
}

\section{The Role of the Hand Surgeon in Microsurgery in Brazil}

\author{
Rosana Raquel Endo ${ }^{1 \odot}$ Carlos Henrique Fernandes ${ }^{2}$ Marcela Fernandes ${ }^{2}$ \\ Joao Baptista Gomes dos Santos ${ }^{2}$ Luiz Carlos Angelini ${ }^{3}$ Luis Renato Nakachima ${ }^{2}$ \\ 1 Serviço de Cirurgia de Mão e Microcirurgia, Hospital do Servidor \\ Público Municipal, São Paulo, SP, Brasil \\ ${ }^{2}$ Departamento de Ortopedia e Traumatologia, Escola Paulista de \\ Medicina, Instituto de Cirurgia da Mão, Universidade Federal de São \\ Paulo, São Paulo, Brasil \\ ${ }^{3}$ Disciplina de Anatomia da Ortopedia, Universidade Metropolitana \\ de Santos, Santos, SP, Brasil \\ Rev Bras Ortop 2019;54:309-315.

\begin{abstract}
Address for correspondence Rosana Raquel Endo, Serviço de Cirurgia de Mão e Microcirurgia, Hospital do Servidor Público Municipal, Rua Castro Alves, 60 - Aclimação, São Paulo, SP 01532-000, Brasil (e-mail: drarosanaendo@gmail.com).
\end{abstract}

\section{Resumo}

\section{Palavras-chave}

- mãos/cirurgia

- reimplante

- procedimentos microcirúrgicos

- fatores socioeconômicos
Objetivo Avaliar quais as condições que o cirurgião de mão no Brasil tem encontrado na prática clínica para a realização de procedimentos microvasculares.

Métodos Pesquisa clínica primária prospectiva, observacional, transversal e analítica; realizada no $37^{\circ}$ Congresso Brasileiro de Cirurgia de Mão, de 30 de março a 1 de abril de 2017, em Belo Horizonte. Por meio de aplicação de questionário a médicos do Congresso, com 12 perguntas, respostas objetivas, fechadas ou de múltipla escolha; envolveram a região geográfica, o tipo de instituição, se pública e/ou privada, seu treinamento microcirúrgico, tempo de formação, condições técnicas, presença de equipe de retaguarda para urgências e remuneração.

Resultados Um total de 143 médicos foram entrevistados, 65,7\% atuavam na região sudeste; $13,3 \%$ na região nordeste; $11,9 \%$ na região sul; $6,3 \%$ na região centro-oeste; e $2,8 \%$ na região norte. Do total de cirurgiões, $43,4 \%$, atuavam há $<5$ anos; $16,8 \%$ de 5 a 10 anos; $23,8 \%$ de 10 a 20 anos; e $23 \%$ há > 20 anos. Do total de cirurgiões, 7,0\% não tiveram treinamento em cirurgias microvasculares; $63,6 \%$ realizaram treinamento na residência médica, 30,8\% em outra instituição, e 7,7\%, outro país. Do total de cirurgiões, 5,6\% trabalhavam em hospitais públicos, 14,7\% em hospitais privados, e $76,9 \%$ em ambos. Do total de cirurgiões, $1,8 \%$ consideravam adequada a remuneração nas instituições públicas e 5,0\% nas instituições privadas; $98,2 \%$ consideraram inadequadas as remunerações nas instituições públicas e 95,0\% nas instituições privadas. Conclusão A maioria obteve treinamento em microcirurgia, não fazia reimplantes, considerava a remuneração inadequada, e não dispunha de equipe de sobreaviso. Há escassez e má distribuição de cirurgiões de mão com habilidade microcirúrgica nas emergências e baixo valor de reembolso.

\footnotetext{
Trabalho feito no Instituto de Cirurgia da Mão, Departamento de Ortopedia e Traumatologia, Escola Paulista de Medicina, Universidade Federal de São Paulo, São Paulo, Brasil.

(1D) Rosana Raquel Endo's ORCID is https://orcid.org/0000-0002-9819301X.
}

received

January 21, 2018

accepted

July 2, 2018
DOI https://doi.org/

10.1055/s-0039-1692433. ISSN 0102-3616.
Copyright $\odot 2019$ by Sociedade Brasileira License terms de Ortopedia e Traumatologia. Published by Thieme Revnter Publicações Ltda, Rio de Janeiro, Brazil 


\begin{abstract}
Objective The present study evaluates which conditions the hand surgeons have found in the Brazilian clinical practices for microvascular procedures.

Methodology A prospective, observational, and analytical primary clinical research conducted during the $37^{\text {th }}$ Brazilian Congress of Hand Surgery from March 30 to April 1, 2017, in Belo Horizonte, to doctors responding 12 closed objective multiple choices questions involving geographic region, type of institution, whether public or private, their microsurgical training, time since graduation, technical conditions, the presence of standby team for emergencies, and their remuneration.

Results The present study analyzed 143 hand surgeons, of which $65.7 \%$ are based in the Southeast region; $13.3 \%$ in the Northeast region; $11.9 \%$ in the South region; $6.3 \%$ in the Central West region; and $2.8 \%$ in the North region. A total of $43.4 \%$ of the hand surgeons had $<5$ years of practice; $16.8 \%$ from 5 to 10 years; $23.8 \%$ from 10 to 20 years; and $23 \%$ had $>20$ years of practice in microvascular surgery. A total of $7.0 \%$ of the surgeons had no training in microvascular surgery; $63.6 \%$ held training in their medical residency, $30.8 \%$ in other institutions; and $7.7 \%$ in another country. A total of $76.9 \%$ of the surgeons worked both in private and public hospitals, $14.7 \%$ in private hospitals, and $5.6 \%$ in public hospitals. Regarding the remuneration, $1.8 \%$ considered it

\section{Keywords}

- hands/surgery

- replantation

- microsurgical procedures

- socioeconomic factors adequate in public hospitals, and $98.2 \%$ considered it inadequate. A total of $5.0 \%$ considered it adequate in private hospitals, and $95.0 \%$ considering it inadequate.

Conclusion The present research has shown that the majority of the surgeons received training in microsurgery, has never performed reimplantation, and considered the remuneration inadequate. There is a lack of a standby team; the distribution of hand surgeons with microsurgical ability in emergencies is scarce and uneven, with low reimbursement value.
\end{abstract}

\section{Introdução}

A mão é a parte do corpo que está mais exposta a traumas. ${ }^{1,2}$ Lesões traumáticas na mão e no punho representam uma das maiores causas de atendimentos nos setores de emergência dos hospitais em todo o mundo. ${ }^{2}$ No Brasil, não dispomos de dados precisos sobre o número destes atendimentos. O conhecimento da epidemiologia e a identificação da população suscetível a este tipo de trauma pode garantir ao paciente um atendimento inicial eficaz para a recuperação funcional da mão. $\mathrm{O}$ manejo inadequado deste paciente causa um custo importante tanto a ele quanto ao sistema de saúde e previdenciário. Sabemos que, não havendo condições técnicas locais, deve haver condições de transferência do paciente para um centro de referência que disponha de equipamento adequado e de equipe médica qualificada. ${ }^{3}$

Nos casos de lesões graves, é necessária a intervenção de uma equipe de cirurgia da mão bem treinada e dentro de um tempo ideal, pois é neste momento que as chances de êxito são maiores. ${ }^{4,5}$

Em certas ocasiões, este atendimento exige conhecimento e treinamento em técnica microcirúrgica. Atualmente, as técnicas microcirúrgicas não estão mais limitadas somente ao tratamento de lesões traumáticas agudas e de amputações de extremidades, elas também englobam procedimentos reconstrutivos, como os retalhos microcirúrgicos. ${ }^{6}$
A justificativa da presente pesquisa é que o bom resultado de um reimplante ou de um retalho microcirúrgico depende tanto das características do paciente e da lesão quanto da disponibilidade de instrumentais adequados, microscópio cirúrgico, destreza e treinamento do cirurgião, assim como de equipe hospitalar capacitada. ${ }^{6,7}$

Até mesmo nos países mais desenvolvidos economicamente, os procedimentos microvasculares realizados por médicos especialistas estão restritos aos centros de trauma, aos hospitais acadêmicos e aos hospitais de atendimento terciário. ${ }^{8}$

O objetivo da presente pesquisa foi avaliar quais as condições que o cirurgião de mão no Brasil tem encontrado na prática clínica para a realização de procedimentos microvasculares.

\section{Material e Métodos}

A presente pesquisa clínica foi realizada mediante aplicação de questionário a médicos participantes do $37^{\circ}$ Congresso Brasileiro de Cirurgia de Mão, em Belo Horizonte, de 30 de março a 1 de abril de 2017.

Tratou-se de uma pesquisa clínica primária prospectiva, observacional, transversal e analítica. ${ }^{9} \mathrm{O}$ questionário aplicado consistiu de 12 perguntas objetivas de fácil entendimento e de respostas objetivas, tipo fechadas, com intuito de aumentar a taxa de resposta conforme a - Fig. $\mathbf{1}$. 


\section{QUESTIONÁRIO}

Atuação do Médico Cirurgião de Mão em Microcirurgia no Brasil

1. Em qual região exerce sua atividade de trabalho?
( ) Sul ( ) Sudeste ( ) Norte
( ) Nordeste ( ) Centro oeste

2. Há quanto tempo atua na especialidade de cirurgia da mão?
( ) até 5 anos ( ) 5-10 anos
( ) 10-20 anos ( ) mais de 20 anos

3. Atua em serviço público e/ou privado?
( ) Público ( ) Privado ( )
Público/Privado

4. Como foi realizado o seu treinamento em procedimentos microvasculares?
( ) Não houve treinamento ( ) No próprio serviço
( ) Em outro país
( ) Em outra instalação
Em caso afirmativo, responda:

5. Você faz reimplante de membros no seu serviço?

Hospital público ( ) Sim ( ) Não

Hospital privado ( ) Sim ( ) Não

6. Você faz retalhos microcirúrgicos no seu serviço?

Hospital público ( ) Sim ( ) Não

Hospital privado ( ) Sim ( ) Não
7. Estes mesmos procedimentos são realizados por outras equipes/especialistas?

Reimplante ( ) Sim ( ) Não

Retalho ( ) Sim ( ) Não

Quanto aos seus locais de trabalho, responda as seguintes perguntas.

8. Há microscópio, instrumental e fios de sutura apropriados para realizar reimplantes e retalhos?

Hospital público ( ) Sim ( ) Não

Hospital privado ( ) Sim ( ) Não

9. Há equipe de sobreaviso de reimplante?

Hospital público ( ) Sim ( ) Não

Hospital privado ( ) Sim ( ) Não

Em caso afirmativo, responda:

10. Os cotos de amputação a serem reimplantados vêm adequadamente acondicionados?

( ) $\operatorname{Sim}($ ) Não

11. E em tempo hábil?
( ) Sim ( ) Não

12. Você considera as tabelas de remuneração adequadas para este procedimento de acordo com a sua região?

Hospital público ( ) Sim ( ) Não

Hospital privado ( ) Sim ( ) Não

Fig. 1 Questionário: Atuação do Médico Cirurgião de Mão em Microcirurgia no Brasil.

Foram coletados dados envolvendo a região geográfica brasileira onde o cirurgião atuava, além do tipo de instituição onde trabalhava, se pública e/ou privada. Foram coletados dados quanto ao treinamento microcirúrgico durante a residência médica, tempo de formação, quais procedimentos microvasculares realizava, presença de condições técnicas, presença de equipe de retaguarda nos casos de urgência e a opinião quanto à remuneração.

Para minimizar o viés, foi enfatizado o anonimato da pesquisa. 
O presente trabalho foi aprovado pela Comissão de Ética e Pesquisa e registrado na Plataforma Brasil com CAAE $n^{\circ}$ 70235417.3.0000.5442.

Os dados das respostas foram submetidos a análises estatísticas utilizando os softwares SPSS Statistics for Windows, Versão 20.0 (IBM Corp. Armonk, NY, EUA), Minitab 16 (Minitab, LLC., State College, PA, EUA) e Microsoft Excel 2010 (Microsoft Corporation, Redmond, WA, EUA) de forma a calcular o teste de igualdade de duas proporções para caracterizar a distribuição da frequência relativa em todas as perguntas.

Para análise estatística dessa pesquisa, definimos um nível de significância de 0,05 (5\%). Todos os intervalos de confiança (ICs) construídos ao longo do trabalho foram construídos com $95 \%$ de confiança estatística.

A análise estatística caracterizou a distribuição de frequência relativa (percentuais) de todas as perguntas do questionário, e para isso foi utilizado o teste de igualdade de duas proporções.

\section{Resultados}

Questão número 1: foram obtidas 143 respostas de médicos cirurgiões de mão quanto à região geográfica de atuação. Do total de cirurgiões, $94(65,7 \%)$ atuavam na região sudeste (referência), 19 (13,3\%) na região nordeste, $17(11,9 \%)$ na região sul, $9(6,3 \%)$ na região centro-oeste, e $4(2,8 \%)$ na região norte. Não foi encontrada significância estatística $(p<0,001)$ ( - Tabela 1).

Questão número 2: quanto ao tempo de atuação na especialidade, $62(43,4 \%)$ cirurgiões (referência) responderam atuarem há $<5$ anos, $24(16,8 \%)$ de 5 a 10 anos, 34 $(23,8 \%)$ de 10 a 20 anos, e $23(16,1 \%)$ há > 20 anos. Não foi encontrada significância estatística ( $p<0,001)$.

Questão número 3: quanto à gestão hospitalar de atuação, $8(5,6 \%)$ cirurgiões responderam trabalhar somente em hospitais públicos, 21 (14,7\%) somente em hospitais privados, e 110 (76,9\%), (referência) em ambos. Não foi encontrada significância estatística $(p<0,001)$.

Questão número 4: durante a residência médica em cirurgia da mão, 10 (7,0\%) cirurgiões não tiveram treina-
Tabela 1 Distribuição por região de atuação dos médicos avaliados

\begin{tabular}{|l|l|l|l|}
\hline $\begin{array}{l}\text { Regiâo na qual exerce } \\
\text { atividade de trabalho }\end{array}$ & $\boldsymbol{n}$ & $\%$ & valor-p \\
\hline Centro-oeste & 9 & $6,3 \%$ & $<0,001$ \\
\hline Nordeste & 19 & $13,3 \%$ & $<0,001$ \\
\hline Norte & 4 & $2,8 \%$ & $<0,001$ \\
\hline Sudeste & 94 & $65,7 \%$ & Ref. \\
\hline Sul & 17 & $11,9 \%$ & $<0,001$ \\
\hline Total & 143 & & \\
\hline
\end{tabular}

mento em procedimentos microvasculares, 91 (63,6\%) realizaram treinamento no próprio serviço de residência médica, 44 (30,8\%) em outra instituição, e 11 (7,7\%) em outro país. Não foi encontrada significância estatística $(p<0,001)$.

Questão número 5: Nos hospitais públicos, 45 (34,9\%) cirurgiões responderam que realizavam reimplantes de membros, e $44(65,1 \%)$ não realizavam. Já nos hospitais privados, $57(45,6 \%)$ realizavam reimplantes de membros, e 68 (54,4\%) não realizavam (-Fig. 2). Apesar desta diferença, não encontramos significância estatística.

Questão número 6: nos hospitais públicos, 80 (63,0\%) cirurgiões afirmaram que realizavam retalhos microcirúrgicos, e 47 (37,0\%) não realizavam. Nos hospitais privados, 75 cirurgiões $(58,6 \%)$ realizavam retalhos microcirúrgicos, e 54 $(41,4 \%)$ não. Esta diferença não apresentou significância estatística.

Questão número 7: de acordo com 34 cirurgiões (27,4\%) entrevistados, há profissionais de outra especialidade que não cirurgiões de mão que realizam reimplantes. Já 90 $(72,6 \%)$ cirurgiões afirmaram que os reimplantes não são realizados por outra especialidade. Quanto aos retalhos, 68 $(50,4 \%)$ cirurgiões responderam que estes são feitos por outra especialidade, e 67 (49,6\%) não são. Esta diferença não apresentou significância estatística.

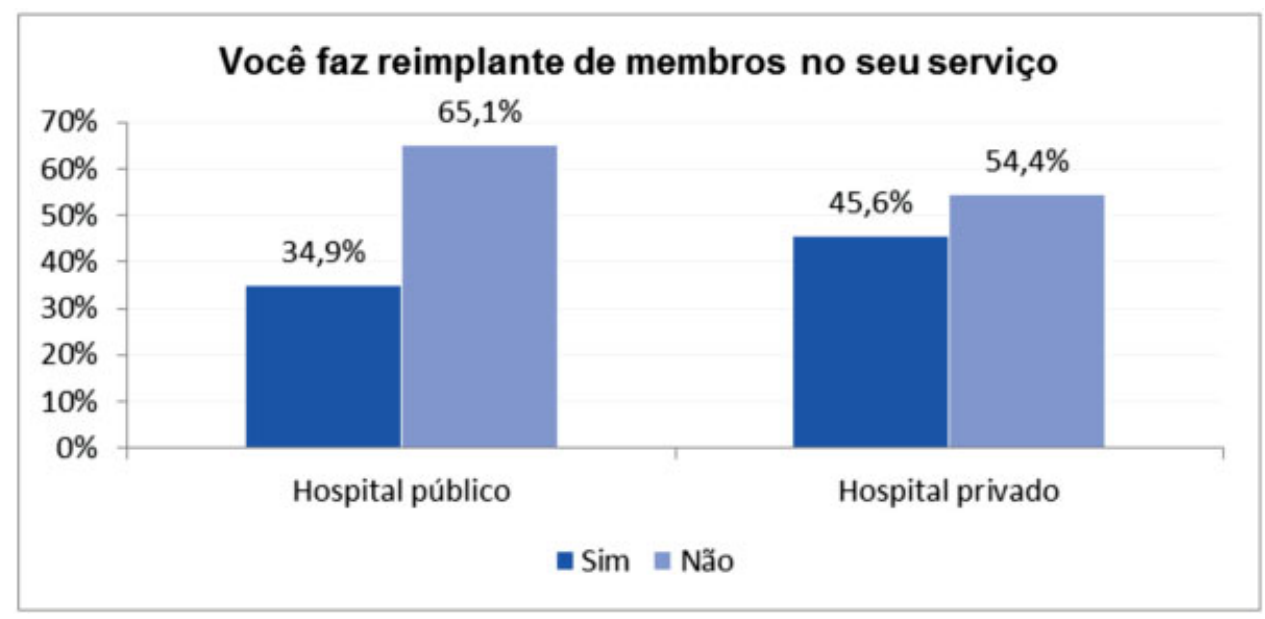

Fig. 2 Realização de reimplante de membros. 
Questão número 8: dos cirurgiões que trabalham em hospitais públicos, 84 (66,1\%) afirmaram dispor de microscópio, instrumental e fios de sutura apropriados para realizar microcirurgias, e 43 (33,9\%) afirmaram não dispor de condições técnicas para realizar os procedimentos. Dentre os cirurgiões que atuam nos hospitais privados, 117 (80,1\%) dispunham adequadamente destes materiais, e 29 (19,9\%) não houve significância estatística.

Questão número 9: sobre a presença de equipe de sobreaviso para reimplante, 22 (19,0\%) cirurgiões responderam que há equipe de sobreaviso nos hospitais públicos, e 94 (81,0\%) responderam que não há. Já nos hospitais privados, 35 (26,5\%) cirurgiões responderam que há equipe de sobreaviso, e 97 (73,5\%) responderam que não há. Não houve significância estatística.

Questão número 10: com relação às condições de condicionamento nas quais os dedos amputados chegam ao hospital, para $38(36,9 \%)$ dos entrevistados, os dedos chegam adequadamente condicionados, e para 65 (63,1\%) dos entrevistados, os dedos não chegam de forma adequada. Não houve significância estatística.

Questão número 11: quanto ao tempo no qual os dedos amputados chegam ao hospital em condições ainda viáveis, 53 (53,0\%) cirurgiões responderam receber em tempo hábil, enquanto $47(47,0 \%)$ responderam que isso não ocorre. No entanto, esta diferença não apresentou significância estatística.

Questão número 12: em relação à remuneração de acordo com a região que atuam, $2(1,8 \%)$ cirurgiões responderam considerá-la adequada nas instituições públicas, e 6 (5,0\%) responderam considerá-la adequada nas instituições privadas, enquanto $112(98,2 \%)$ as consideraram inadequadas nas públicas e 114 (95,0\%) nas privadas, porém esta diferença não apresentou significância estatística.

\section{Discussão}

Pesquisas são comumente utilizadas para avaliar o uso e o resultado de novas práticas clínicas para tratamento de doenças. Os dados obtidos podem influenciar nas políticas de saúde coletiva e auxiliar na construção de protocolos. ${ }^{9}$

O questionário é uma ferramenta de coleta de dados constituída por uma série ordenada de perguntas, que devem ser respondidas por escrito e sem a presença do entrevistador. A presente pesquisa foi feita mediante aplicação de questionário anônimo impresso durante um congresso nacional da especialidade de cirurgia da mão, composta de 12 questões de fácil leitura e interpretação e de facilidade na resposta, pelo método de múltipla escolha. Não foi possível obter resposta de todos os participantes, devido às dificuldades de acesso aos congressistas em um local amplo; uma minoria não apresentou interesse em responder ao questionário após a apresentação do tema.

No levantamento bibliográfico, não foi encontrado nenhum artigo com esta mesma metodologia para a avaliação da atuação do cirurgião de mão com técnicas microcirúrgicas.

Sobre este mesmo assunto, encontramos 3 trabalhos cuja metodologia de pesquisa foi feita por questionário eletrônico e que apresentaram taxa de resposta entre 18,5 e $45 \%$. 7,8,10 Encontramos dois trabalhos que utilizaram pesquisa via telefone e a taxa de resposta variou de 85 a 86\%. ${ }^{11}$ Em um estudo, os autores utilizaram o formato eletrônico e, caso não conseguissem resposta, o contato foi feito por telefone. Desta forma, houve $100 \%$ de taxa de resposta, porém o número do público alvo era bem menor que nos trabalhos anteriores. ${ }^{6}$

A maior taxa de resposta neste questionário foi obtida por cirurgiões que atuam há < cinco anos na especialidade. Nossos resultados foram diferentes dos encontrados na literatura, cuja maioria ativa compõe o período entre 9 e 20 anos de atuação. ${ }^{12,13}$

Semelhante ao que ocorre fora do país, a disposição geográfica dos médicos entrevistados apresentou uma distribuição de razão semelhante ao volume populacional por região no país, estando a maioria no sudeste, seguido pelo nordeste, as duas regiões brasileiras mais populosas, respectivamente. $^{14}$

De acordo com o questionário, 76,9\% dos cirurgiões atuam em hospitais privados e públicos, 14,7\% somente em hospitais privados, e $5,6 \%$ somente em hospitais públicos. Apesar de possuir um sistema de saúde diferente do brasileiro, trabalhos envolvendo os membros da sociedade americana de cirurgia da mão mostraram que a maioria dos cirurgiões também atua em instituições privadas, sendo 48,4\% em hospitais particulares sem vínculo acadêmico, e 18,8\% com filiação acadêmica. ${ }^{7,10}$

Por meio de pesquisa realizada em instituições americanas cadastradas como referência para realizar procedimentos microvasculares, foi verificado que $47 \%$ dos centros de trauma nível I dispunham de acesso imediato ao cirurgião de mão e possibilidade para reimplante de forma contínua e definitiva. E, quando analisados os centros de trauma nível II, esta taxa caiu para $29 \%{ }^{11}$

A maior taxa de resposta no questionário da presente pesquisa foi obtida por cirurgiões que atuam há $<5$ anos na especialidade. Nossos resultados foram diferentes do encontrados na literatura, cuja maioria ativa compõe o período entre 9 e 20 anos de atuação. ${ }^{12,13}$

A maioria dos entrevistados desta pesquisa teve treinamento durante a residência médica, e somente $7 \%$ dos entrevistados relataram não terem tido treinamento durante a formação.

O constante treinamento feito de forma apropriada poderá aprimorar as técnicas microcirúrgicas independente de qual for a especialidade de base. ${ }^{15}$

Nos EUA, apesar de $70 \%$ dos cirurgiões considerarem excelente o treinamento em microcirurgia durante a residência, grande parte optou por não realizar reimplante. As justificativas foram: estarem com os horários ocupados por procedimentos eletivos e prática clínica (51\%), perda da confiança na habilidade cirúrgica (39\%) e baixo retorno financeiro por um procedimento considerado longo e de alta complexidade (30\%). ${ }^{7,8,16}$

Acreditamos que nossos resultados mostram que tal tendência se repete no Brasil, visto que a maioria respondeu não realizar reimplantes, com significância estatística quando relacionado a hospitais públicos. 
Já quanto ao procedimento de retalhos microcirúrgicos, a taxa de reposta foi positiva em $63 \%$ dos entrevistados nas instituições públicas. Apesar de a literatura fazer referência de que na prática privada os retalhos microcirúrgicos ainda são economicamente viáveis, não obtivemos diferença significativa entre a proporção de cirurgiões que os fazem dos que não os fazem na rede privada. ${ }^{17}$

Apesar da maioria dos cirurgiões entrevistados ter tido formação em microcirurgia durante a formação, a qual abrange treinamento para realização de retalhos microcirúrgicos, isto não parece se refletir na prática do exercício profissional. Estes resultados foram semelhantes aos achados norte-americanos, que demonstram que para $94 \%$ dos cirurgiões, a prática microcirúrgica representa $<25 \%$ dos seus procedimentos. ${ }^{8,18}$

Quanto ao retalho, na presente pesquisa, foi observado que $50,4 \%$ dos cirurgiões de outra especialidade o fazem em comparação aos cirurgiões de mão. No entanto, esta diferença não foi estatisticamente significante.

Nos EUA, o dado que se tem é de que cirurgiões de mão que primeiramente fizeram residência em cirurgia plástica foram mais propensos a realizar procedimentos microvasculares gerais, reimplantes e retalhos livres do que os cirurgiões treinados em residência ortopédica. ${ }^{70} 10$ Mesmo assim, a frequência de procedimentos microvasculares entre cirurgiões plásticos nos EUA vem diminuindo, e isso se deve principalmente ao baixo retorno financeiro. ${ }^{19}$

Com relação à presença de equipe de reimplante nos hospitais em território nacional, observamos ausência de $81 \%$ na rede pública e de $73,5 \%$ na rede privada.

Dados literários norte-americanos sobre os fatores institucionais pertinentes ao manejo do paciente com trauma de mão apontam falhas, como escassez de especialistas capacitados para realizar atendimento de emergência no local do acidente e falta de protocolos para orientar a real necessidade de transferência para os grandes centros. ${ }^{20}$

Mais de $95 \%$ dos cirurgiões entrevistados consideraram inadequada a tabela de valores para os procedimentos microcirúrgicos, um dos fatores que possivelmente explica o desestímulo para esta atuação, similar ao que ocorre nos EUA., ${ }^{7,8}$

$\mathrm{O}$ treinamento em microcirurgia faz parte da grade curricular nos programas de residência médica para a especialidade de cirurgia da mão. ${ }^{20-27}$ Porém, ainda é um questionamento que devemos fazer se este treinamento é suficiente para uma boa prática e se o substrato que foi dado ao médico durante sua formação lhe dá aptidão e segurança suficientes para realizar todos os procedimentos após o término da graduação.

Não ter tido uma boa base é um forte preditor para este profissional não exercer a especialidade na sua plenitude. ${ }^{7}$

Em nosso estudo, a maioria dos participantes, tanto de instituições pública quanto privadas, responderam não realizar reimplantes, assim como outros profissionais, diferentemente do retalho microcirúrgico, o qual a maioria dos cirurgiões de mão e outros profissionais apontaram exercer.

Mesmo sendo as lesões traumáticas de mão e punho uma boa parcela das procuras nos prontos atendimentos, $81 \%$ dos cirurgiões entrevistados indicaram não haver equipe de sobreaviso para reimplante na rede pública, e 73,5\% indica- ram a ausência deste tipo de equipe na rede privada apesar, da maioria dos hospitais terem os materiais e equipamentos adequados e apenas $7 \%$ dos entrevistados não terem tido treinamento microcirúrgico.

Há evidencias de que vem diminuindo o número de reimplantes nos últimos 10 anos, reflexo de uma melhor capacitação do cirurgião em selecionar e melhor indicar tal procedimento e da diminuição de casos de amputação devido ao aprimoramento das medidas de segurança implementadas no ambiente de trabalho, local onde epidemiologicamente ocorre a maioria das lesões traumáticas na mão. ${ }^{8,16}$

Fatores como o baixo retorno financeiro, consumo de tempo, ausência de colegas qualificados para auxiliar durante a cirurgia, ausência de equipe capacitada na enfermaria para o manejo pós-operatório correto e crescentes implicações médico-legais são alguns pontos encontrados na literatura como limitantes. $6,8,19,27$

Para manter o refinamento da técnica microcirúrgica, que é crucial para um resultado satisfatório, o cirurgião precisa praticar constantemente. No entanto, fatores educacionais, financeiros e práticos parecem não colaborar para isso.

Os custos do treinamento em laboratórios são onerosos e necessitam de elevada carga horária, ${ }^{15}$ e os próprios procedimento microvasculares costumam ter longa duração, tempo este do qual muitos cirurgiões não dispõem, seja ele tomado por cirurgias eletivas ou por considerarem que isto não contribua para uma boa qualidade de vida.

Mais de $95 \%$ consideraram inadequada a tabela de valores para estas cirurgias, outro fator que possa explicar o desestímulo para esta atuação.

A maioria dos entrevistados teve treinamento durante a formação; alguns realizaram mais de um curso em outra instituição e fora do país, mas esta alta taxa de treinamento não parece influenciar a prática clínica.

As lesões traumáticas da mão são sensíveis ao tempo e requerem cuidados altamente especializados a fim de assegurar não somente o salvamento de um dígito e sim também da sua funcionalidade. ${ }^{20,21,27}$ Sendo assim, se o caso for mal conduzido logo no início, ele estará fadado ao fracasso e isto trará prejuízos tanto para o paciente quanto para o sistema de saúde, justamente por este paciente não poder mais trabalhar por um período prolongado e necessitar de mais intervenções e reabilitações para obter um resultado minimamente satisfatório, ou podendo ficar incapacitado permanentemente. ${ }^{11,16,23}$

Embora não tenha havido uma relevância estatística, significando que não houve uma diferença estatística entre as comparações, isto não anulou as comparações das questões analisadas; uma sugestão da autora é que novas pesquisas possam ser realizadas, em outros congressos, grupos de estudos e seminários nacionais de cirurgias de mão, com outros grupos de médicos especialistas nesta área, para que se possam obter resultados mais seguros de acordo com as questões analisadas.

\section{Conclusão}

A distribuição geográfica dos cirurgiões da mão no Brasil mostrou-se proporcional à densidade demográfica por 
região do país, concentrando-se os especialistas nas regiões mais populosas. A maioria deles obteve treinamento em microcirurgia, atua em hospitais públicos e privados, realiza retalhos microcirúrgicos, porém não faz reimplantes e considera a remuneração inadequada.

Não há outras especialidades que façam reimplantes, e a maioria dos hospitais não dispõe de equipe de sobreaviso para reimplante, embora possuam materiais adequados.

Há muito que melhorar tendo em vista as tendências decrescentes para reimplante e procedimentos microvasculares, devido à escassez e à má distribuição de cirurgiões de mão com habilidade microcirúrgica nos setores de emergência e o baixo valor de reembolso.

\section{Conflitos de Interesses}

Os autores declaram não haver conflitos de interesses.

\section{Referências}

1 Hing E, Hall MJ, Ashman JJ, Xu J. National Hospital Ambulatory Medical Care Survey: 2007 outpatient department summary. Natl Health Stat Rep 2010;23(28):1-32

2 Trybus M, Lorkowski J, Brongel L, Hladki W. Causes and consequences of hand injuries. Am J Surg 2006;192(01):52-57

3 de Putter CE, Selles RW, Polinder S, Panneman MJ, Hovius SE, van Beeck EF. Economic impact of hand and wrist injuries: health-care costs and productivity costs in a population-based study. J Bone Joint Surg Am 2012;94(09):e56

4 del Piñal F. Severe mutilating injuries to the hand: guidelines for organizing the chaos. J Plast Reconstr Aesthet Surg 2007;60(07): 816-827

5 Pomerance J, Truppa K, Bilos ZJ, Vender MI, Ruder JR, Sagerman SD. Replantation and revascularization of the digits in a community microsurgical practice. J Reconstr Microsurg 1997;13(03):163-170

6 Christensen TJ, Anding W, Shin AY, Bishop AT, Moran SL. The Influence of Microsurgical Training on the Practice of Hand Surgeons. J Reconstr Microsurg 2015;31(06):442-449

7 Elliott RM, Baldwin KD, Foroohar A, Levin LS. The impact of residency and fellowship training on the practice of microsurgery by members of the american society for surgery of the hand. Ann Plast Surg 2012;69(04):451-458

8 Payatakes AH, Zagoreos NP, Fedorcik GG, Ruch DS, Levin LS. Current practice of microsurgery by members of the American Society for Surgery of the Hand. J Hand Surg Am 2007;32(04): 541-547

9 Hochman B, Nahas FX, Oliveira Filho RS, Ferreira LM. Desenhos de pesquisa. Acta Cir Bras 2005;20(02, Suppl 2):2-9

10 Mehta K, Pierce P, Chiu DT, Thanik V. The effect of residency and fellowship type on hand surgery clinical practice patterns. Plast Reconstr Surg 2015;135(01):179-186

11 Peterson BC, Mangiapani D, Kellogg R, Leversedge FJ. Hand and microvascular replantation call availability study: a national real- time survey of level-I and level-II trauma centers. J Bone Joint Surg Am 2012;94(24):e185

12 Gaspar MP, Kane PM, Honik GB, Shin EK, Jacoby SM, Osterman AL. Geographic and Age-Based Variations in Medicare Reimbursement Among ASSH Members. Hand (N Y) 2016;11(03): 347-352

13 Waljee JF, Zhong L, Shauver MJ, Chung KC. The influence of surgeon age on distal radius fracture treatment in the United States: a population-based study. J Hand Surg Am 2014;39(05): 844-851

14 Instituto Brasileiro de Geografia e Estatítica, Departamento de População e Indicadores Sociais, Divisão de Estudos e Análises da Dinâmica Demográfica. Projeto, Fundo de População das Nacões Unidas no Brasil (BRA/98/P08), Sistema Integrado de Projeções e Estimativas Populacionais e Indicadores Sóciodemográficos. Dsiponível em: https://ww2.ibge.gov.br/home/estatistica/populacao/projecao.../publicacao_UNFPA.pdf

15 Vinagre G, Villa J, Amillo S. Microsurgery Training: Does It Improve Surgical Skills? J Hand Microsurg 2017;9(01):47-48

16 Davas Aksan A, Durusoy R, Ada S, Kayalar M, Aksu F, Bal E. Epidemiology of injuries treated at a hand and microsurgery hospital. Acta Orthop Traumatol Turc 2010;44(05):352-360

17 Wade JW, Stephens JA, Bragham RF, Carbo JD. Microsurgery in private practice: is it feasible economically? Ann Plast Surg 2001; 46(03):255-259, discussion 259-260

18 Lerman OZ, Haddock N, Elliott RM, Foroohar A, Levin LS. Microsurgery of the upper extremity. J Hand Surg Am 2011;36(06): 1092-1103, quiz 1103

19 Chen MW, Narayan D. Economics of upper extremity replantation: national and local trends. Plast Reconstr Surg 2009;124(06): 2003-2011

20 Maroukis BL, Chung KC, MacEachern M, Mahmoudi E. Hand Trauma Care in the United States: A Literature Review. Plast Reconstr Surg 2016;137(01):100e-111e

21 Christoforou D, Alaia M, Craig-Scott S. Microsurgical management of acute traumatic injuries of the hand and fingers. Bull Hosp Jt Dis (2013) 2013;71(01):6-16

22 Wolfe VM, Wang AA. Replantation of the upper extremity: current concepts. J Am Acad Orthop Surg 2015;23(06):373-381 Review

23 Soucacos PN. Indications and selection for digital amputation and replantation. J Hand Surg [Br] 2001;26(06):572-581

24 Breahna A, Siddiqui A, Fitzgerald O'Connor E, Iwuagwu FC. Replantation of digits: a review of predictive factors for survival. J Hand Surg Eur Vol 2016;41(07):753-757

25 Brown M, Lu Y, Chung KC, Mahmoudi E. Annual Hospital Volume and Success of Digital Replantation. Plast Reconstr Surg 2017;139 (03):672-680

26 Mahmoudi E, Swiatek PR, Chung KC. Emergency Department Wait Time and Treatment of Traumatic Digit Amputation: Do Race and Insurance Matter? Plast Reconstr Surg 2017;139(02): $444 \mathrm{e}-454 \mathrm{e}$

27 Sears ED, Chung KC. Replantation of finger avulsion injuries: a systematic review of survival and functional outcomes. J Hand Surg Am 2011;36(04):686-694 